\title{
Calcarea carbonica derivative complex (M8) as adjuvant treatment of inflammatory mammary carcinoma in a dog
}

\author{
Daniella Matos da Silva, Eneida Janiscki Da Lozzo, Carolina Camargo de \\ Oliveira, Dorly de Freitas Buchi, Simone Domit Guérios.
}

Federal University of Paraná, Brazil.

\begin{abstract}
Background: Inflammatory mammary carcinoma (IMC) is locally aggressive, fast growing, highly malignant tumor that affects humans and dogs. Affected dogs usually are presented with generalized edema, pain, erythema, and skin ulceration in mammary glands. Surgery is not recommended and an effective treatment has not been established [1]. Calcarea carbonica derivative complex (M8) has demonstrated anticancer properties in a murine model, by improving innate immune response against tumor cells [2,3]. M8 is a complex high diluted medication comprised of a 10\%-20\% concentration of Calcarea carbonica, Aconitum napellus, Arsenicum album, Asa foetida, Conium maculatum, Ipecacuanha, Phosphorus, Rhus tox, Silicea, Sulphur, and Thuya occidentalis, all in decimal dilutions of Hahnemann in distilled water and submitted to vigorous shaking.
\end{abstract}

Aim: Describe an association of M8 and piroxicam (Non-steroidal anti-inflammatory drug) to treat a dog with IMC.

Discussion: A 7 years old, mixed breed intact female dog was presented to the Federal University of Parana Veterinary Hospital, Curitiba (HV-UFPR) for mammary glands examination. The owners related inflammation of mammary glands with clinical course of approximately 10 days, which was treated for mastitis (cephalexin and metergoline) without clinical improvement. Clinical examination revealed erythema, increased skin warmth, pain on palpation, and plaque involving the $4^{\text {th }}$ and $5^{\text {th }}$ right mammary glands. Abdominal ultrasound and serum biochemistry were unremarkable. Thoracic radiographs showed suspicious images of pulmonary metastasis. Fine needle biopsy was taken for cytologic examination. Cytological interpretation was a malignant epithelial neoplasm, probably a mammary carcinoma. Diagnosis of IMC was based on clinical signs and cytopathology. Dog was treated with oral $(0.5 \mathrm{~mL})$ and topical M8 twice a day for 15 days, and pyroxican, $0.3 \mathrm{mg} / \mathrm{kg}, \mathrm{PO}, \mathrm{q} 24 \mathrm{~h}$. Clinical improvement was observed 7 days after starting treatment. Until present date (70 treatment days with M8), dog has no clinical signs of IMC, and does not show signs of disease progression.

Conclusion: The present report suggests that M8 associated with piroxicam contributes to improvement of IMC dog's quality of life and survival rate. However, further clinical studies are needed to evaluate response to treatment in patients diagnosed with IMC.

Keywords: Calcarea carbonica complex; inflammatory mammary carcinoma; piroxicam 


\title{
References
}

[1] Sorenmo K. Canine mammary gland tumors. Veterinary Clinics of North America: Small Animal Practice. 2003;33(3):573-96.

[2] Guimaraes F, Abud A, Oliveira S, Oliveira C, Cesar B, Donatti L, et al. Stimulation of lymphocyte antimelanoma activity by co-cultured macrophages activated by complex homeopathic medication. BMC Cancer. 2009;9:293.

[3] Guimaraes F, Andrade L, Martins S, Abud A, Sene R, Wanderer C, et al. In vitro and in vivo anticancer properties of a Calcarea carbonica derivative complex (M8) treatment in a murine melanoma model. BMC Cancer. 2010;10(1):113.

\section{Calcarea carbonica e associações (M8) como tratamento adjuvante de carcinoma inflamatório mamário em cão}

\begin{abstract}
RESUMO
Introdução: O carcinoma inflamatório mamário (CIM) é um tumor altamente maligno que acomete cães e pessoas, apresentando-se localmente invasivo e com crescimento rápido. Em cães, os sinais clínicos incluem edema e eritema generalizado das mamas acometidas, dor local e ulceração. A intervenção cirúrgica é contraindicada e não há consenso sobre tratamento clínico eficaz [1]. Estudos em modelo murino demonstraram que Calcarea carbonica e associações (M8) possuem propriedades anticancerígenas através de estímulo da resposta imune inata [2,3]. O M8 é altamente diluído, composto de 10 a 20\% de Calcarea carbonica, Aconitum napellus, Arsenicum album, Asa foetida, Conium maculatum, Ipecacuanha, Phosphorus, Rhus tox, Silicea, Sulphur, e Thuya occidentalis, todos na diluição decimal de Hahnemann em água destilada e submetido à agitação vigorosa.
\end{abstract}

Objetivo: Descrever a associação de M8 e piroxicam (antiinflamatório não esteroidal) no tratamento de cão com CIM.

Discussão: Uma cadela não castrada, sem raça definida, de 7 anos de idade foi trazida ao Hospital Veterinário da Universidade Federal do Paraná - Curitiba (HV-UFPR) com histórico de inflamação mamária com evolução de 10 dias e não responsiva ao tratamento para mastite (com cefalexina e metergolina). Ao exame físico, as mamas abdominais caudais e inguinais direita apresentavam-se em placa, com aumento de temperatura, edema e eritema localizados e presença de sensibilidade dolorosa ao toque. A ultrassonografia abdominal e bioquímica sérica não apresentaram alterações significativas, enquanto que a radiografia torácica evidenciou imagem sugestiva de metástase pulmonar. Realizou-se biópsia aspirativa por agulha fina para análise citológica, a qual foi compatível com neoplasia epitelial maligna, provavelmente carcinoma mamário. O diagnóstico de CIM baseou-se nos sinais clínicos e resultados citopatológicos. Instituiu-se tratamento com M8 oral ( $0,5 \mathrm{~mL}$ a cada 12 horas) e tópico (nas mamas envolvidas), em associação com piroxicam $(0,3 \mathrm{mg} / \mathrm{kg}$, PO, a cada 24 horas). Observou-se melhora clínica significativa após 7 dias de tratamento e até a presente data (70 dias de tratamento com M8) a paciente não apresenta sinais clínicos de CIM e de progressão da doença. 
Conclusão: O presente caso sugere que a associação de M8 e piroxicam contribui para melhora da qualidade de vida e aumento da taxa de sobrevida em cães com CIM. No entanto, mais estudos são necessários para avaliar a resposta clínica de pacientes com CIM tratados com M8.

Palavras chave: Calcarea carbônica; carcinoma inflamatório mamário; piroxicam

\section{(c)) BY-NC-ND Licensed to GIRI}

Support: authors declare that this study received no funding

Conflict of interest: authors declare there is no conflict of interest

Correspondence author: Eneida Janiscki Da Lozzo, eneidaja@uol.com.br

How to cite this article: da Silva DM, Da Lozzo EJ, de Oliveira CC, Buchi DF, Guérios SD. Calcarea carbonica derivative complex (M8) as adjuvant treatment of inflammatory mammary carcinoma in a dog. Int J High Dilution Res [online]. 2011 [cited YYYY Month dd]; 10(36): 268-270. Proceedings of the XXV GIRI Symposium and VIII CBFH; 2011 Sep 04-07; Foz do Iguaçu (Brazil). GIRI and ABFH; http://www.feg.unesp.br/ ojs/index.php/ijhdr/article/view/468/479 